\title{
An online radon monitor for low-background detector assembly facilities
}

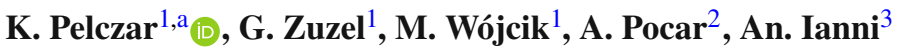 \\ ${ }^{1}$ M. Smoluchowski Institute of Physics, Jagiellonian University, Kraków, Poland \\ 2 Amherst Center for Fundamental Interactions and Physics Department, University of Massachusetts, Amherst, MA 01003, USA \\ ${ }^{3}$ Physics Department, Princeton University, Princeton, NJ 08544, USA
}

Received: 6 July 2020 / Accepted: 23 December 2020 / Published online: 27 January 2021

(C) The Author(s) 2021

\begin{abstract}
Backgrounds from long-lived radon decay products are often problematic for low-energy neutrino and rareevent experiments. These isotopes, specifically ${ }^{210} \mathrm{~Pb},{ }^{210} \mathrm{Bi}$, and ${ }^{210} \mathrm{Po}$, easily plate out onto surfaces exposed to radonloaded air. The alpha emitter ${ }^{210} \mathrm{Po}$ is particularly dangerous for detectors searching for weakly-interacting dark matter particles. Neutrons produced via $(\alpha, n)$ reactions in detector materials are, in some cases, a residual background that can limit the sensitivity of the experiment. An effective solution is to reduce the ${ }^{222} \mathrm{Rn}$ activity in the air in contact with detector components during fabrication, assembly, commissioning, and operation. We present the design, construction, calibration procedures and performance of an electrostatic radon detector made to monitor two radon-suppressed clean rooms built for the DARKSIDE-50 experiment. A dedicated data acquisition system immune to harsh operating conditions of the radon monitor is also described. A record detection limit for ${ }^{222} \mathrm{Rn}$ specific activity in air achieved by the device is $0.05 \mathrm{mBqm}^{-3}$ (STP). The radon concentration of different air samples collected from the two DARKSIDE50 clean rooms measured with the electrostatic detector is presented.
\end{abstract}

\section{Introduction}

Events resulting from the attachment of ${ }^{222} \mathrm{Rn}$ progeny onto the surfaces of detectors searching for rare processes, such as solar neutrinos and particle dark matter, can be the limiting backgrounds of current and future experiments. The long-lived isotopes ${ }^{210} \mathrm{~Pb}\left(\mathrm{~T}_{1 / 2}=22.3 \mathrm{y}\right),{ }^{210} \mathrm{Bi}\left(\mathrm{T}_{1 / 2}=5 \mathrm{~d}\right)$, and ${ }^{210} \mathrm{Po}\left(\mathrm{T}_{1 / 2}=138 \mathrm{~d}\right)$ are particularly dangerous because they accumulate onto detector components exposed to air throughout the construction, commissioning, and operation

a e-mail: krzysztof.pelczar@gmail.com (corresponding author) of the experiments and cannot be let decay away in a reasonable time. Generally, dedicated low-background procedures need to be implemented to limit these backgrounds to acceptably low levels.

Surface alpha background, such as ${ }^{210} \mathrm{Po}$, can be a dominant contributor to the background for direct dark matter experiments. These can contribute to background through a few distinct mechanisms. Alpha decays, usually easily identifiable when they occur in the bulk of detectors by their relative scintillation and ionization share and scintillation time profile, can mimic the low energy deposition of weakly interacting dark matter particles if their energy is sufficiently degraded, as is possible when the decays happen on surfaces. The alpha decay of ${ }^{210} \mathrm{Po}$ produces a nucleus of ${ }^{206} \mathrm{~Pb}$ with about $100 \mathrm{keV}$ of kinetic energy, which can be mistaken with a target nucleus recoiling from a weakly interacting massive particle (WIMP) when the alpha particle is missed, as is the case for about $50 \%$ of surface decays. These two surface backgrounds are identifiable and handily discriminated in detectors able to spatially identify them as surface events. Neutrons from $(\alpha, n)$ reactions occurring on the innermost surfaces of detectors, however, cannot be effectively tagged via spatial cuts, due to the long range and capture time of neutrons. Thus this source of background has to ultimately be contained by directly minimizing its presence on detector materials.

Radon daughters plate out on components of a detector can be reduced and even virtually eliminated by suppressing the radon content in air where the detector is assembled, installed, and commissioned. Equally important is to monitor the radon content in radon-suppressed environments with adequate sensitivity, typically $1 \mathrm{mBq} \mathrm{m}^{-3}$ and in real time. The most sensitive techniques for ${ }^{222} \mathrm{Rn}$ assay (currently reaching detection limits of $\approx 30 \mu \mathrm{Bq}$ ) employ ultra lowlevel proportional counters [1] and cryogenic detectors [2,3]. The latter allow the concurrent detection of ${ }^{220} \mathrm{Rn}$. However, 
neither method is suitable for continuous monitoring. Lucas cell scintillation chambers are a commonly-employed technology, but due to relatively high background count rates, their sensitivity is limited to about $0.5 \mathrm{~Bq} \mathrm{~m}^{-3}$ [4], insufficient for today's needs in rare-event physics.

Alternatively, ${ }^{222} \mathrm{Rn}$ can be detected using the electrostatic collection of radon daughters produced in a large volume of air in which a strong electrostatic field is applied onto the sensitive surface of an $\alpha$-particle detector. The measurement of the consecutive $\alpha$ decays of ${ }^{218} \mathrm{Po}$ and ${ }^{214} \mathrm{Po}$ allows, when properly calibrated, to measure the radon concentration in the sampled air. Radon monitors employing this method were successfully developed by the Super-Kamiokande [5, 6] and BOREXINO [7] experiments. The quoted sensitivity is $1.4 / 0.7 \mathrm{mBq} \mathrm{m}^{-3}\left({ }^{222} \mathrm{Rn}\right.$ in water $)$, and $1 \mathrm{mBq} \mathrm{m}^{-3}$, respectively, adequate for what was required for the DarkSide-50 time projection chamber (TPC). A radon detector based on this technique was built for continuous monitoring of the air of the two clean rooms in which the DarkSide-50 TPC was assembled and commissioned. Our design was supported by Monte Carlo simulations used to optimize the drift electric field and maximize the collection efficiency of radon daughters onto a silicon detector. In addition, signal efficiency was aided by the use of real-time pulse shape discrimination of the alpha signals. For easy efficiency calibrations we made a dedicated dry ${ }^{222} \mathrm{Rn}$ source in the form of stainless steel disc with ${ }^{226}$ Ra electrodeposited on its surfaces.

\section{The DARKSIDE-50 clean rooms}

The first ${ }^{222} \mathrm{Rn}$-suppressed clean room $\left(<1 \mathrm{~Bq} \mathrm{~m}^{-3}\right.$ of ${ }^{222} \mathrm{Rn}$ in air) was designed, built, and operated at Princeton University in 1998-2001 for the construction of the BOREXINO nylon vessels. Thanks to the radon-reduced environment and other low-background procedures, the surface alpha activities of the nylon vessels was below $10 \mathrm{~m}^{-2}$ day $^{-1}[8,9]$.

The DARKSIDE Collaboration built two radon-suppressed clean rooms for the DARKSIDE-50 (DS-50) experiment, and operated them in Hall C of Laboratori Nazionali del Gran Sasso: Clean Room Hanoi (CRH) and Clean Room One (CR1). The clean rooms were used for detector assembly and initial installation, and for subsequent upgrades of the Time Projection Chamber. Their make-up air was entirely supplied by a custom-fitted radon abatement system able to deliver sub-mBqm${ }^{-3}$ air. Monitoring of radon content in the air was crucial for the control of the intrinsic detector background. Radon content in the air (and other technology gases like nitrogen and argon) was measured online by a dedicated electrostatic radon monitor, described in detail in this paper.

The CRH is located on top of the Counting Test Facility (CTF) tank hosting the DARKSIDE-50 Water Cherenkov Veto (WCV) muon detector to which it allows direct access via a flanged opening on its floor. Access is also possible into the DARKSIDE-50 Liquid Scintillator Veto (LSV) detector housed inside a spherical stainless steel tank surrounding the TPC through a gate valve placed approximately $6 \mathrm{~m}$ above the center of the LSV [10]. The CRH houses the cryogenic and liquid argon handling system and the front-end electronics for all sub-detectors (TPC, neutron veto, muon veto). The CR1 clean room houses the equipment used for precision cleaning, preparation, and partial assembly of the DS-50 LAr-TPC parts. It also houses a large vacuum evaporator in which the inner surfaces of the TPC were coated with TPB wavelength shifter. In DS-50, surface contamination was significantly minimized by carrying out all final operations for TPC assembly in highly ${ }^{222} \mathrm{Rn}$-suppressed air clean rooms: CR1 for precision cleaning of the detector parts, wavelength shifter coating, and pre-assembly of the photomultiplier tubes, and CRH for the final assembly, cabling, and insertion into the cryostat of the TPC. In order to take full advantage of the radon-filtration performance of the radon abatement system, both CR1 and CRH were built with radon mitigation included at the design phase. Air handlers and main clean rooms were designed to operate in overpressure with respect to Hall $\mathrm{C}$, great care was placed in sealing the rooms for uncontrolled leaks, such as at feedthrough patch panels, and all surfaces, walls and floors, were lined entirely with stainless steel panels to limit the internal ${ }^{222} \mathrm{Rn}$ emanation. In addition to the usual clean room procedures, staff members entering $\mathrm{CR} 1$ and $\mathrm{CRH}$ are required to station in air-shower vestibules via software-controlled interlocks for long enough to allow sufficient lung volume air exchange and flushing of the air volumes mixed with outside air with low-radon air before access to the main rooms.

\section{Radon abatement system}

Both DARKSIDE-50 clean rooms, supplied with ${ }^{222} \mathrm{Rn}$ (and ${ }^{220} \mathrm{Rn}$ ) depleted air, were instrumental in meeting the background requirements of DARKSIDE-50. In turn, the processed Hall $\mathrm{C}$ air (after radon filtration) had to contain less than $1 \mathrm{mBq} \mathrm{m}^{-3}$ of ${ }^{222} \mathrm{Rn}$. The typical concentration of ${ }^{222} \mathrm{Rn}$ in Hall $\mathrm{C}$ is $\sim 100 \mathrm{~Bq} \mathrm{~m}^{-3}$. The desired radon reduction factor was therefore $>10^{5}$.

Figure 1 shows a schematic view of the radon abatement system designed and delivered by the Ateko company [11]. This system is similar to that described in Ref. [12], although significant DARKSIDE-specific improvements to the control software were implemented.

Ambient Hall $\mathrm{C}$ air is compressed and delivered to the system at a pressure of 8 to 10 bar. In a first stage of processing, the air is dried on an adsorption bed, where its dew point is reduced to $-70^{\circ} \mathrm{C}$ and carbon dioxide is also eliminated. Here it is separated from oil vapors and undergoes micro- 


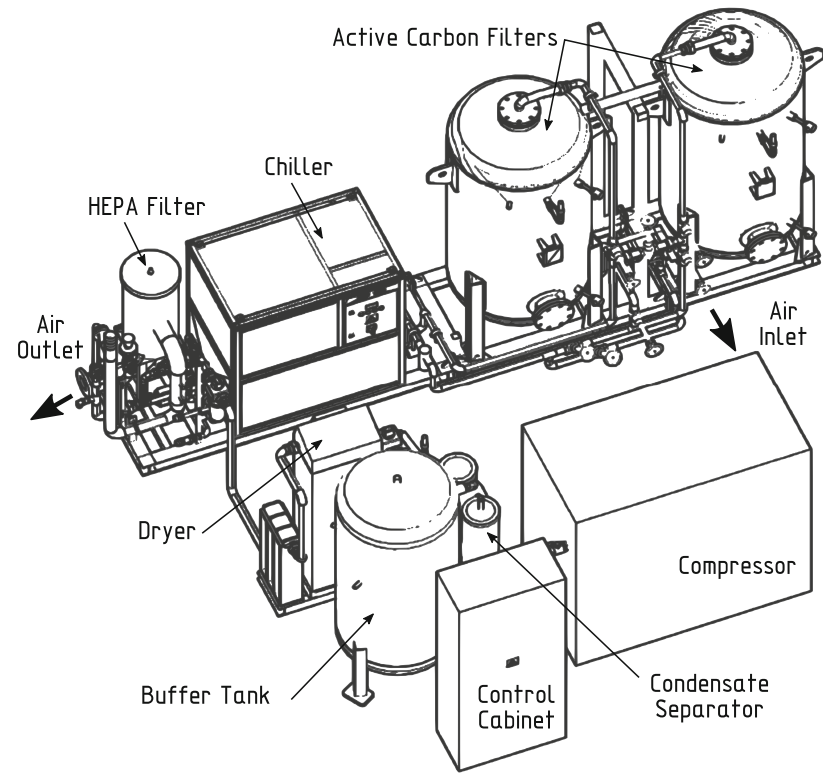

Fig. 1 Schematic view of the DARKSIDE-50 radon abatement system installed in Hall C of LNGS (by courtesy of Ateko)

filtration. Air emerging from the chiller unit at a temperature between $-58{ }^{\circ} \mathrm{C}$ to $-63^{\circ} \mathrm{C}$ is fed to low-temperature activated charcoal-filled radon adsorption columns. The Ateko system has two charcoal columns and can be operated with one adsorption column, two adsorption columns in parallel, or both in series, depending on the radon reduction factor or air throughput required. Normal DARKSIDE-50 operation of the system is with the two adsorption columns in series and limited throughput, for maximal radon suppression. The radon-free air (Ateko air) emerging from the charcoal beds, is fed through a HEPA dust particle filter, then warmed up to $20^{\circ} \mathrm{C}$, and fed to the DS-50 clean rooms. The yield of the system is $\sim 200 \mathrm{~m}^{3} \mathrm{~h}^{-1}$ of radon-suppressed air.

\section{Online electrostatic radon monitor}

In order to constantly monitor the radon content in air produced by the Ateko radon abatement system and air present in the CRH and CR1 clean rooms, a dedicated online electrostatic radon monitor was designed, installed, and commissioned. In addition to air, the system was also calibrated for sampling argon and nitrogen used by the DARKSIDE-50 experiment.

\subsection{Simulations}

The shape of the radon detector vessel was chosen to achieve the following goals: simplified production, minimized weld length and maximized volume-to-surface ratio (i.e., as spherical as possible, in order to minimize the internal ${ }^{222} \mathrm{Rn}$ ema- nation) and maximized geometrical efficiency of ion collection in the electric field of the vessel.

We have simulated known shapes of the detectors used e.g. in the Gerda experiment [7], or detectors made of standard vacuum parts like wide ConFlat pipe assemblies. The figure of merit used to evaluate the detector designs was the number of ions' paths (starting point generated uniformly inside the vessel) ending at the detector's active surface within a certain amount of time (less than $5 \mathrm{~s}$ [13]). In the case of the new detector, we chose the vessel made of three parts: two convex end caps each made of one piece of metal, and a short cylinder to join them.

A full 3D model of the detector was created, and the electric field was numerically calculated using dedicated software [14]. The simulated electric field is shown in Fig. 2. Radon daughter ions are positive and follow a negative potential gradient to the silicon alpha diode. We varied vertical position of the diode observing the maximum electric field strength at its perimeter (not to exceed the breakdown voltage of air and nitrogen). Most of the electric field lines $(\approx 90 \%)$ terminate on the alpha particle detector active surface, with only a small fraction ending on its housing, yielding an expected high collection efficiency for radon daughters. Only small volumes close to the inlet and outlet ports, or located directly above the alpha particle detector, are excluded from ion collection. Detailed model of the diode construction allowed us to investigate the ion paths ending on its active surface. It is important to note here, that the Ortec Ultra-AS alpha detector used in our setup has an elevated rim around the active surface, which in certain conditions may effectively block ions from the large volume of the detector (if placed too "deep" into the volume of the vessel).

Additionally, the drift of radon daughters was also modeled in detail including diffusion of ions in the gas (Fig. 3). We concluded that the diffusion processes do not affect the collection efficiency significantly, ${ }^{1}$ as the collection of ions in the electric field is rather fast, with drift times on the order of seconds $[13,15]$, during which the diffusion of ions is limited.

\subsection{Detector construction}

As discussed above, our main goal was to construct a lowbackground and real-time ${ }^{222} \mathrm{Rn}$ detector, based on electrostatic collection of the charged radon daughters. In order to reach the assumed detection limit the following requirements had to be fulfilled:

\footnotetext{
${ }^{1}$ Radon progenies could enter or escape dead volumes of the detector vessel due to their diffusion in gas.
} 


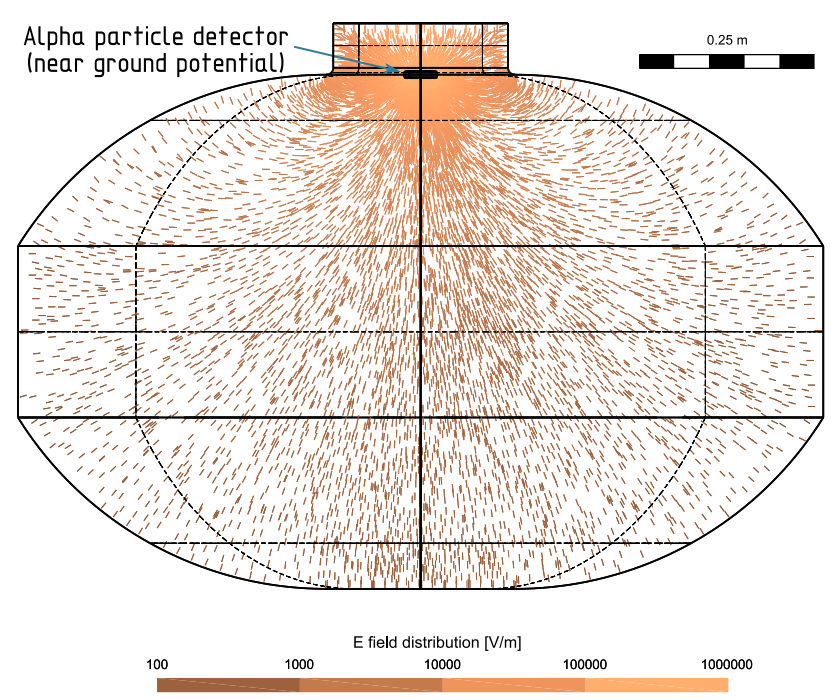

Fig. 2 Electric field distribution inside the radon detector (with $8 \mathrm{kV}$ applied to the vessel) - most of the field lines $(\approx 90 \%)$ close on the Ortec diode assuring high collection efficiency of the charged radon daughters. Breakdown voltage of various gases that could be assayed under normal (or slightly below) atmospheric pressure, is not reached anywhere in the detector volume (for air it is $3 \mathrm{MV} \mathrm{m}^{-1}$, nitrogen $3.45 \mathrm{MV} \mathrm{m}^{-1}$ )

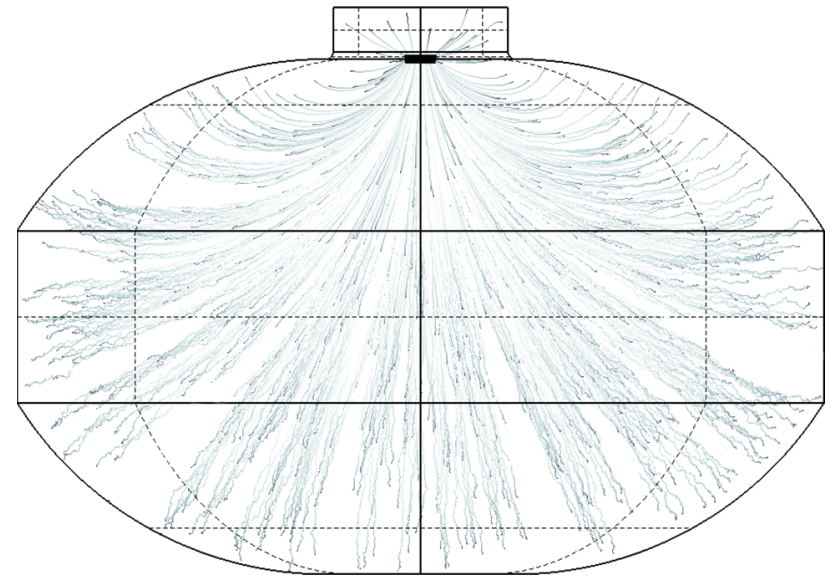

Fig. 3 Visualization of ${ }^{214}$ Po trajectories in the detector vessel. Particle diffusion is included in the computation. Overall, the geometrical collection efficiency (due to the electric field shape) is estimated to be $\approx 90 \%$. Typical ion drift time towards the alpha detector is below $2 \mathrm{~s}$. The detector is therefore capable of measuring ${ }^{220} \mathrm{Rn}$ activity as well

- detector vessel volume in the range of $450 \mathrm{~L}$, the shape optimized for maximal collection efficiency of the radon daughters,

- only stainless steel, copper and PTFE (as an insulator) used as construction materials (proven to have low ${ }^{222} \mathrm{Rn}$ emanation rates),

- all internal metal surfaces electropolished,

- construction of the vessel (certified as a pressure tank) with minimal length of welds (known source of radon),

- all-metal construction: connections/joints metal sealed ( $\frac{1}{2}$ " VCR fittings, ConFlat flanges),
- usage of a round (to prevent discharges) low-background and large-surface alpha detector to register alpha decays of the ${ }^{222} \mathrm{Rn}$ daughters,

- positive bias voltage applied to the detector chamber should be optimized taking into account the collection efficiency of ions and avoiding discharges (the goal is operation with voltages $<10 \mathrm{kV}$ ).

We selected an Ortec Ultra-AS alpha detector, displaying excellent energy resolution despite its large sensitive area. Its active surface is $1200 \mathrm{~mm}^{2}$, with a depletion depth of about $100 \mu \mathrm{m}$ (minimizing muon contribution to the background) and a background count rate in the range of some tens of counts per day (cpd) in the energy range of $1.5 \mathrm{MeV}-$ $10 \mathrm{MeV}$. The diode is operated at $40 \mathrm{~V}$, but due to a conductive entrance contact (500 ̊ thick) the detector does not produce external electric field which could disturb the collection of the positively charged radon daughters. ${ }^{2}$ The main advantage of the Ortec detector is its shape (no sharp edges) and large active surface area. The large active area allows for easier electric field lines guidance towards the detector. Also, larger dimensions of the detector result in smaller gradients of the electric field close to the detector edges.

The detector vessel with a diameter of $1 \mathrm{~m}$ and height of $70 \mathrm{~cm}$ (volume of $450 \mathrm{~L}$ ) was made from stainless steel and electropolished inside. Its shape is shown in Fig. 3. A schematic of the radon monitor setup is shown in Fig. 4. The alpha detector is installed on the main top flange (DN CF-250) using a dedicated PTFE holder such that its active surface is exactly at the level of the top curvature of the tank. In addition, the connection of the flange neck to the tank is appropriately rounded. Internals of the detector vessel do not have any sharp edges to minimize the electric field gradients. The detector signal feedthrough and the gas outlet were welded in the top flange. The gas inlet was connected to the bottom flange (DN CF-40). Since high voltage is applied to the vessel, the inlet and the outlet lines had appropriate electrically-insulating ceramic insertions. The support legs of the monitor rest on large PTFE pads (approximately $10 \mathrm{~cm}$ high).

The high voltage applied to the detector vessel is provided by a dedicated high-high voltage (HHV) unit (Bertan 225$30 \mathrm{R}$ ) able to deliver up to $20 \mathrm{kV}$ with very low ripple (400 $\mathrm{mV}$ ), effectively eliminating this internal source of electronic noise.

The detector was installed in a dedicated protection box equipped with a control panel as shown in Fig. 4. The panel allows for selection of a gas to be assayed and for calibrations with a dedicated dry ${ }^{222} \mathrm{Rn}$ emanation source. In the

${ }^{2}$ In detectors discussed in [5,7] Si-PIN diodes with negative bias were used. This configuration was more favorable for collection of positively charged ions. 


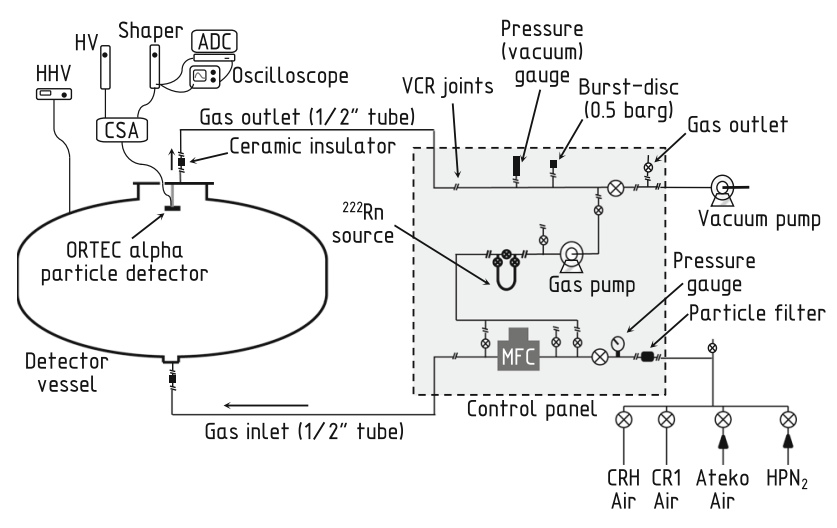

Fig. 4 Schematic of the DARKSIDE radon online monitor

latter case, circulation of the gas is enforced by a small pump operating in a closed loop, including the calibration source and the main vessel. While measuring air, its flow rate is controlled by a mass flow controller (MFC). Before entering the detector the air is additionally filtered with a Swagelok sintered metal filter. To control the pressure at the inlet and in the detector vessel, one analog and one digital vacuum/pressure gauges were installed. In the configuration shown in Fig. 4 one can measure the radon-reduced air, transferred directly from the radon abatement system (the Ateko air) or from the clean rooms CRH and CR1.

An additional line is connected to the BOREXINO highpurity nitrogen $\left(\mathrm{HPN}_{2}\right)$ system, which is practically radonfree - its ${ }^{22} \mathrm{Rn}$ content is below $1 \mu \mathrm{Bqm}^{-3}$ [16]. $\mathrm{HPN}_{2}$ was used to flush the detector and measure its background. Ateko air and $\mathrm{HPN}_{2}$ are available at high pressure of about 7 bar. The gas lines connected with CRH and CR1 are not pressurized, therefore the air has to be additionally sucked through the detector by a dedicated vacuum pump installed at the detector outlet (see Fig. 4). A burst disc ( 0.5 bar) prevents a sudden and uncontrolled pressure increase, which could damage the system. In the construction of the detector vessel and the control panel, only full-metal components (valves, tubing) and metal-sealed joints/flanges (VCR, CF) were used in order to reduce the internal ${ }^{222} \mathrm{Rn}$ emanation.

\subsubsection{Signal readout}

The radon monitor is operated in harsh environmental conditions. The analogue signal readout path is therefore susceptible to electronic noise pickup, despite the full metal box enclosure and use of an additionally filtered, low noise, high voltage power supply. The electronic noise may come from the high power electrical equipment installed in the Hall $\mathrm{C}$ of LNGS (like powerful fluorescent lights, heavy-load cranes, proximity to the radon abatement system, air conditioning systems, etc.), or residual micro-discharges occurring in the vessel. The noise sources usually induce fake pulses. Their shapes differ from the shapes of signals induced by alpha decays. They often have spikes, a leading or trailing negative undershoot or are followed by a poorly shaped after-pulse.

Vetoing of the invalid pulses is difficult using analogueonly components. Some techniques have been proposed to veto such events [17], but they require additional readout electronics, often interfacing the high voltage electrode of the detector vessel. A simple and inexpensive method of pulse shape digitization and analysis was employed in our design, allowing for powerful discrimination between the positive signals coming from alpha decays and fake pulses generated by the noisy environment. Additionally, the method does not require any modifications of the existing readout electronics, especially readout of the high voltage signal (i.e., from anode), and thus may be applied directly to existing set-ups as a simple upgrade.

Micro-discharges caused by the high voltage bias of the detector vessel may induce pulses of oscillating character, or exhibit spikes often saturating the preamplifier. Depending on the location of the discharge, or the character of other disturbing events, the rise time of pulses observed at the output of the charge sensitive preamplifier may also be longer, accompanied by other features like spikes, undershoots etc.

Shaping amplifiers are usually designed to produce "semi-Gaussian" pulses for the typical output pulses of the charge-sensitive preamplifiers (producing exponential decay), because their output crudely approximates a Gaussian curve. Any deviation from the semi-Gaussian pulse shape may be an indication of a poorly formed input pulse, that may not originate in a typical interaction of an alpha particle, but due to a limited bandwidth of shaping amplifiers details of (malformed) pulses are often lost. Such pulses shaped by the shaping amplifier without any additional vetoing system, may have energies reconstructed by a Multi Channel Analyzer (MCA) often falling into the range of ${ }^{214} \mathrm{Po}$ or ${ }^{218}$ Po alpha decay energies.

In our setup the Ortec diode is read-out by a single-channel charge-sensitive preamplifier (CAEN A1422), matched to the silicon detector capacitance. Next, the pulses are directly digitized by a dedicated analogue to digital converter (ADC, 12-bit, up to 7.2 Megasamples/s of sampling speed) built in a microcontroller. The data is sent in real time to a computer. The pulse shape digitizer, together with the control software on a PC function as an MCA, producing the energy spectra of the registered alpha particle (after pulse shape discrimination and digital reconstruction of the event energy).

The Pulse Shape Discrimination (PSD) technique applied to the recorded pulses, and used here to distinguish between the proper (valid) pulses and the fake (invalid) pulses, is simple. The output pulses of the charge sensitive amplifier ideally have a sharp leading edge and the exponential decay for the events induced by nuclear interactions (mostly alpha and beta particles). At the same time, majority of the fake 


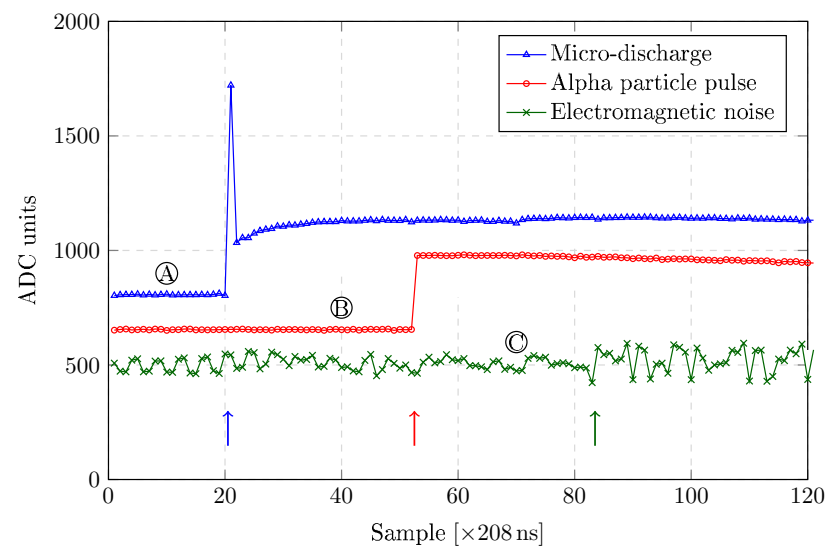

Fig. 5 Charge-sensitive preamplifier pulses recorded by the ADC. A micro-discharge generated pulse (triangles, blue) exhibits a sharp spike on the leading edge, and slow rise time of the following part. A valid pulse (circles, red), of nearly the same reconstructed energy is shown for a comparison. Arrows indicate trigger positions (shifted to ease comparison)

events, generated by the (electronic) environmental noise, depart from the ideal shape, having additional features like under- or overshoots, lower rise times, spikes, induced noise (pick-up), ringing, etc. Figure 5 shows three recorded pulses of different origins for a comparison. As an example, a microdischarge generated pulse exhibits a sharp spike on the leading edge (Fig. 5, trace A), and slow rise time of the following part. A valid pulse of nearly the same reconstructed energy is shown as trace B. An analogue MCA would register both pulses as valid. Additionally, some electromagnetic induced noise pulses (trace C) populate the lower part of the alpha particle energy spectrum. In an analogue analysis path, a high threshold is required to reject such pulses. The labels $A, B, C$ correspond to the plots shown in Fig. 7, where the pulse parameters are mapped to their reconstructed energy and PSD parameter values.

The PSD analysis is based on two signal features extracted from the digitized pulses. Each feature is assigned a properly scaled measure between 0 and 0.5. Overall PSD parameter of an event is a sum of the two values.

The first parameter is simply the pulse rise time at the trigger position. The digitized pulse is low-pass filtered to remove the excess high frequency noise. The filtered pulse is then used to compute the rise time. A typical value of the rise time for valid pulses is on the order of $200 \mathrm{~ns}$, corresponding to one ADC sample (the sampling rate of the ADC used was 4.8 Megasamples/s).

The second pulse shape feature, used in the PSD analysis is based on the comparison of the original pulse shape with the filtered one (low-pass). Firstly, the difference between the two versions of the pulse is calculated yielding a residuum of the pulse. Next, sum of absolute sample values of the residuum is computed and appropriately scaled. The sum values close to 0 correspond to smooth, valid pulses without excess noise or spikes. For pulses having sharp spikes, often caused by micro-discharges, or electromagnetic noise, the sum becomes significant.

Both pulse shape quality measures are scaled to the range $0-0.5$ such that 0.5 in both cases corresponds to an ideal pulse shape induced by an alpha particle (sharp leading edge, no excess noise). The sum of the quality measures yields the PSD parameter for each event falling in the range $0-1$. Next, pulses with the PSD values below a certain threshold are rejected from further analysis, and do not contribute to the alpha particle energy spectrum. Both algorithms are computationally inexpensive, and easily applicable for the analysis of the recorded data.

The cut threshold value was determined experimentally by collecting large statistics calibration data with reduced high voltage (down to $4 \mathrm{kV}$ ) applied to the detector vessel (where the majority of recorded pulses was produced by alpha particles, Fig. 6) and long-term background measurement data with nominal high voltage $(8 \mathrm{kV})$ applied to the detector vessel (Fig. 7). In the latter case a number of recorded pulses clearly exhibited distorted shapes, like spikes or slow rise times. Both pulse groups were then used to determine the upper and lower bounds for the PSD cut parameter: majority of the valid pulses was assigned the PSD parameter above 0.78 , while most of the invalid pulses have values of the PSD parameter below 0.73 . Therefore the PSD cut was arbitrarily set at 0.75 , independent of the pulse energy. It was observed that only a small fraction of valid PSD pulses was rejected by the algorithm (conservatively less than $0.5 \%$ - see single events below the Po lines in Fig. 6), insignificant for an analysis usually bearing a statistical error of $30 \%$ (see Sect. 4.3.3). Therefore the PSD algorithm efficiency is $\epsilon_{P S D} \approx 100 \%$.

The final alpha particle energy spectrum is constructed after application of the PSD cut, thus allowing us to operate the detector with high voltages biasing the vessel, without spectrum distortions from micro-discharges.

\subsubsection{Slow control and monitoring}

The entire system is monitored by dedicated slow control software allowing remote access to all devices, check of their status and change of parameters. Accessible are: the HHV unit, the oscilloscope (for the control of pulse shapes in case of, e.g., problems caused by excess noise), the mass flow controller, the vacuum/pressure gauges and the pulse digitizer. The pulse digitizer records, analyzes and displays the energy spectrum of the detected alpha particles. Another program displays the ${ }^{222} \mathrm{Rn}$ concentration in real-time. Based on the ${ }^{218} \mathrm{Po}$ or ${ }^{214} \mathrm{Po}$ activities, a corresponding ${ }^{222} \mathrm{Rn}$ specific activity is calculated and plotted as a function of time. 


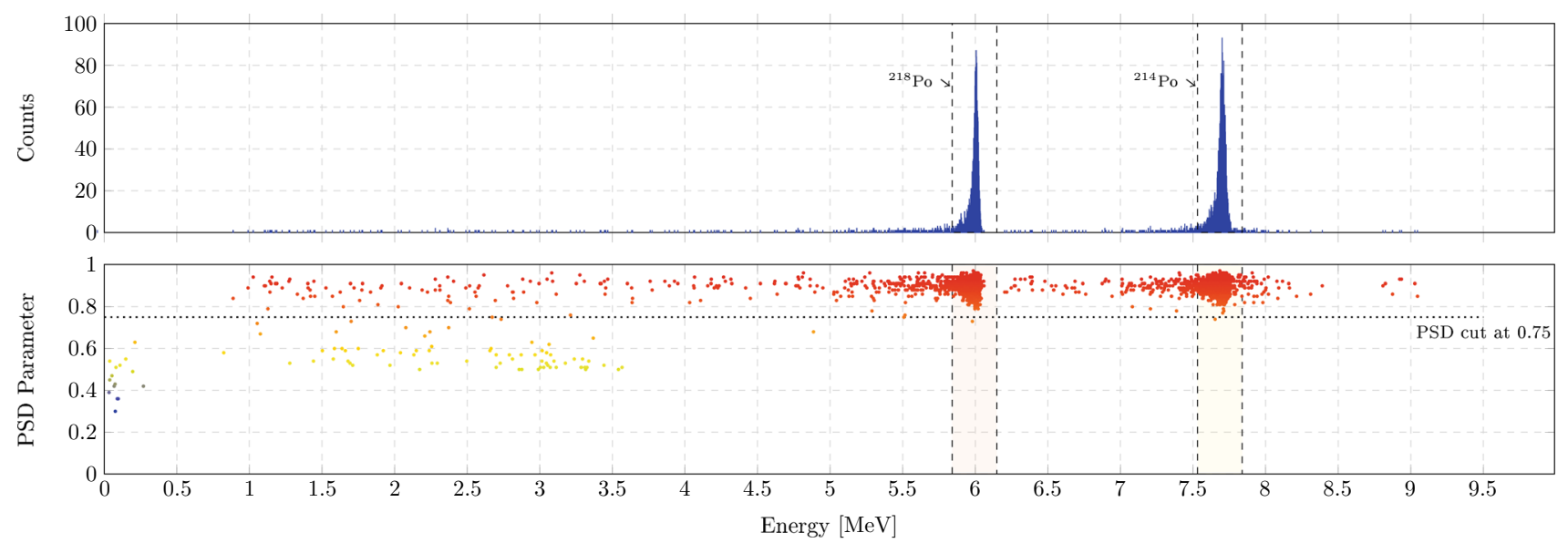

Fig. 6 Calibration data (dry ${ }^{222} \mathrm{Rn}$ source) collected by the ADC, and analyzed by the PSD algorithm. The data was collected at high voltage reduced to $4 \mathrm{kV}$ to minimize the number of invalid pulses reaching the high energy range of the spectrum. Most of the events produced by alpha decays of ${ }^{218} \mathrm{Po}$ and ${ }^{214} \mathrm{Po}$ were assigned a PSD parameter well above 0.75 . The lower part of the energy spectrum was mainly populated by pulses having distorted shape. They were assigned PSD values below the threshold of acceptance $(0.75)$, and thus rejected for the energy spectrum

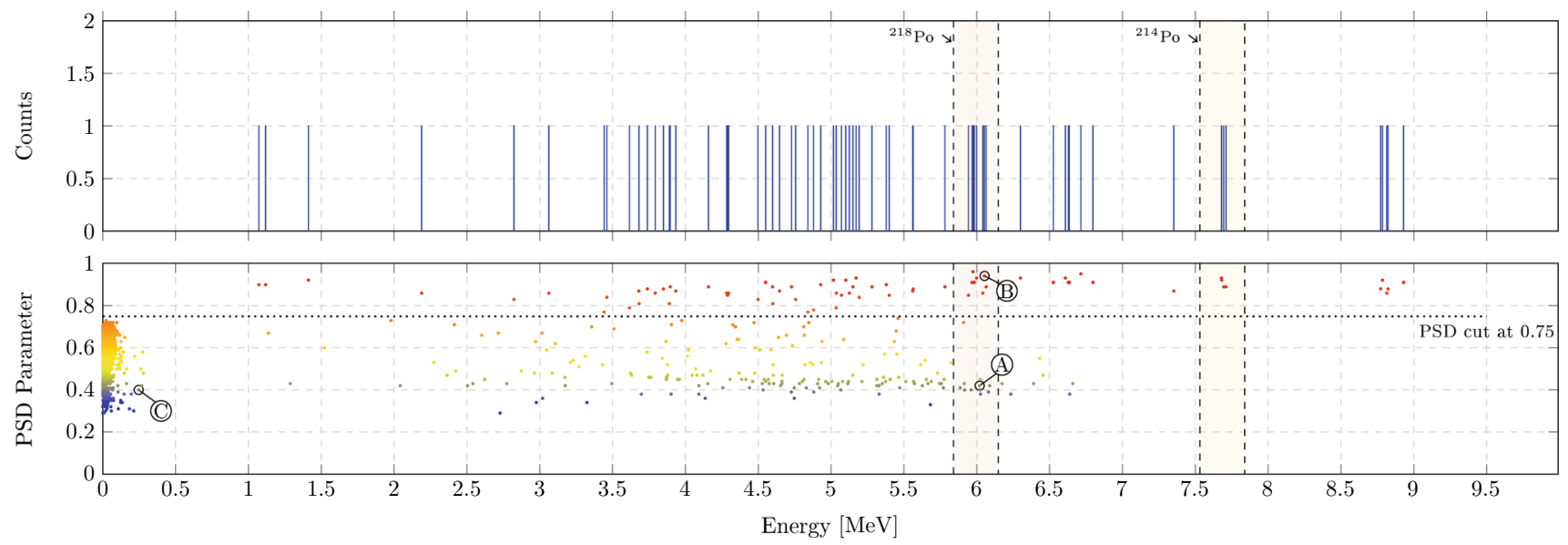

Fig. 7 Example background data collected with high voltage applied to the detector vessel. The high energy range (approximately above 1 $\mathrm{MeV}$ ) is populated by real events stemming from the background activity of the detector and by energetic micro-discharges. For the latter, the PSD analysis yields PSD values well below 0.5 . One of such events is

\subsection{Detector performance}

\subsubsection{Calibration}

A dry calibration source in a form of ${ }^{226} \mathrm{Ra}$ electroplated on a stainless steel disc was permanently connected to the control panel (see Fig. 4). This allowed easy calibration checks by circulating the gas in a closed loop. The activity of the dry calibration source is $(45 \pm 2) \mathrm{mBq}$.

The measurement of the overall detection efficiency was done with the vessel filled with the Ateko air using the built-in ${ }^{222} \mathrm{Rn}$ dry source. This measurement was particularly impor- shown in Fig. 5, labeled on the PSD plot as A. Without the PSD analysis, this type of events would contribute to the detector background in the region of ${ }^{218} \mathrm{Po} 6 \mathrm{MeV}$ alpha line (labeled $B$ ). Events generated by electromagnetic noise, usually having energy below MCA channel 200, are assigned PSD values lower than 0.75 (labeled $C$ )

tant since the ${ }^{218} \mathrm{Po}$ and ${ }^{214} \mathrm{Po}$ ion collection efficiencies depend on the neutralization processes by chemical agents. Presence of water, hydrocarbons and other electronegative molecules in the air sample neutralizes the ions before they may be collected on the alpha detector [18]. Therefore the detection efficiency calibrations were performed in the same experimental conditions as the further measurements.

A ratio $R$ of the ${ }^{214}$ Po to ${ }^{218}$ Po count rates could be used as an indicator of the gas purity [7] and to compare the conditions of different measurements. $\mathrm{R}$ approaches 1 if neutralization of ${ }^{218} \mathrm{Po}$ is rather small and almost all ions reach the alpha detector surface. Else, the ${ }^{214}$ Po count rate is higher 
since there is an additional collection channel of ions within the decay chain between ${ }^{218} \mathrm{Po}$ to ${ }^{214} \mathrm{Bi}$. In our case, the ratio $\mathrm{R}$ of the two Po isotope count rates was always very close to 1 , so ${ }^{222} \mathrm{Rn}$ content in the sampled air was determined directly from the registered Po count rates. We were performing calibrations with the dry source directly before or after the radon assays.

The initial calibration also served two other purposes:

- determination of the optimal high voltage applied to the detector vessel,

- determination of the energy scale for the (digital) multichannel analyzer.

The obtained calibration spectrum (PSD algorithm applied), recorded after the equilibrium between ${ }^{222} \mathrm{Rn}$ and the daughters had been reached $-3 \mathrm{~h}$ after radon injection, is shown in Fig. 8. The ${ }^{218} \mathrm{Po}$ and ${ }^{214} \mathrm{Po}$ peaks are of the same intensities indicating, as expected, air of high purity (the abatement system removes water, $\mathrm{CO}_{2}, \mathrm{CO}$ and particulate matter from the air). The high voltage potential applied to the detector vessel was $8 \mathrm{keV}$. For higher voltages, up to $20 \mathrm{kV}$, the count rate under both Po peaks increased by about $12-15 \%$, but above $9 \mathrm{kV}$ a visible increase of electronic noise has been observed (populating the low-energy part of the spectrum and slightly worsening the detector energy resolution). It was still easily cut-off by the PSD algorithm and setting higher discrimination threshold for the ADC, but taking into account relatively small gain in the count rates we decided to operate the detector at the HHV $=8 \mathrm{kV}, 3$ also not to risk any failures of the semiconductor detector or the HHV system. The overall absolute detection efficiency $(\epsilon)$ for ${ }^{218} \mathrm{Po}$ and ${ }^{214} \mathrm{Po}$ in Ateko air was the same and equal to $(36 \pm 3) \%$. In general, the detection efficiency $\epsilon=\epsilon_{c o l} \epsilon_{g e o} \epsilon_{P S D}$, where $\epsilon_{c o l}$ is the collection efficiency of ions on the active surface of the alpha detector, and $\epsilon_{\text {geo }}$ is the geometrical detection efficiency (probability to register an alpha decay of ion present on the detector's surface). PSD efficiency is $\approx 100 \%$. Since $\epsilon_{\text {geo }} \approx 50 \%$ ( $2 \pi$ geometry), one gets $\epsilon_{\text {col }} \approx 72 \%$. The ion collection efficiency is lower than the estimated $90 \%$ (see Sect. 4.1), as only a fraction of ${ }^{218}$ Po retains charge right after alpha decay of ${ }^{222} \mathrm{Rn}$. Taking the fraction of freshly formed and positively charged ${ }^{218}$ Po equal to approximately $87.3 \%$ [19], the expected efficiency of our setup becomes $78.6 \%$, close to the measured collection efficiency of $72 \%$. Also, some of the ions neutralize before reaching the detector, therefore their decays cannot be registered. Additional calibrations were performed for $\mathrm{HPN}_{2}$ and yielded similar results.

The achieved energy resolution (FWHM) for the $6 \mathrm{MeV}$ peak $\left({ }^{218} \mathrm{Po}\right)$ was better than $40 \mathrm{keV}$, comparable to the man-

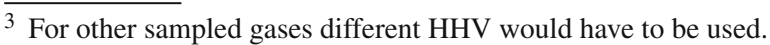

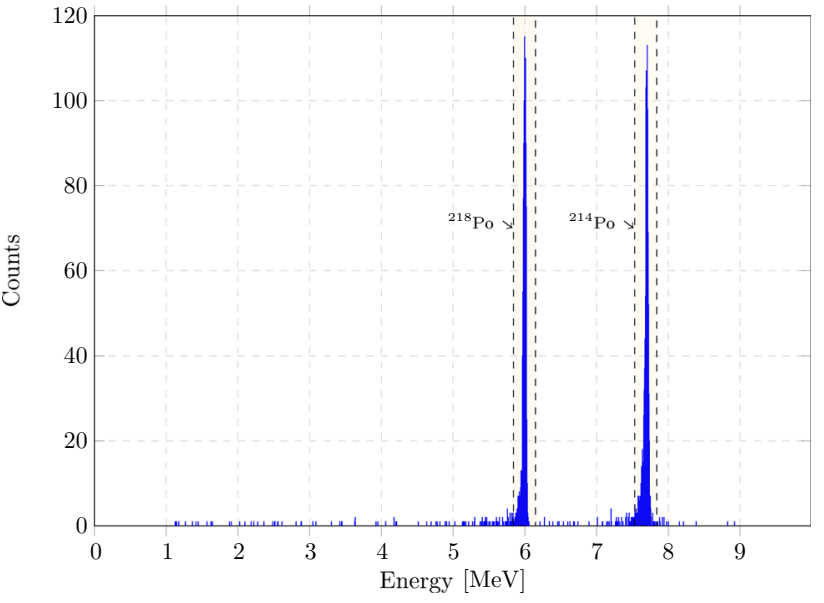

Fig. $8{ }^{222} \mathrm{Rn}$ calibration spectrum (after application of the PSD). ${ }^{214} \mathrm{Po}$ and ${ }^{218}$ Po peaks are of the same intensities. The detector vessel was filled with the purified Ateko air

ufacturer's specification of the diode ( $35 \mathrm{keV}$ for $5.5 \mathrm{MeV}$ alphas). Slightly worse resolution was caused by rather high electronic noise level in Hall C of LNGS, where the detector is operated. Nevertheless the digital energy reconstruction proved to work well on the noisy signals. The resolution allowed to easily resolve all the peaks originating from ${ }^{222} \mathrm{Rn} /{ }^{220} \mathrm{Rn}$ and their daughters (see Fig. 9).

\subsubsection{Background}

After calibration, the detector background was measured for two different operating modes. In the first case, after thorough flushing of the detector vessel with $\mathrm{HPN}_{2}$ it was closed at a slight overpressure (930 mbar, the ambient pressure in Hall $\mathrm{C}$ is about $900 \mathrm{mbar}$ ) and the Po count rates were recorded over time, as shown in Fig. 10. The observed increase during almost 4 weeks (plotted for ${ }^{218} \mathrm{Po}$ ) is a result of the buildup of radon emanated from the detector vessel and other components (the fitted curve reflects the ${ }^{222} \mathrm{Rn}$ in-growth time) with the saturation value of $(61.6 \pm 1.0) \mathrm{cpd}$, corresponding to an absolute activity of $(2.0 \pm 0.2) \mathrm{mBq}$. This value is higher than expected (e.g. from scaling up the blank value of $0.15 \mathrm{mBq}$ of an $80 \mathrm{~L}$ full-metal electropolished emanation chamber [1], one gets about $0.8 \mathrm{mBq}$ ), but the mounting of the detector was not done in the best possible conditions (e.g. in a clean room). The same concerns construction of the gas control panel, which happened in an open space in LNGS Hall C.

The second measurement was performed during continuous flushing of the detector with high purity nitrogen at the rate of $5 \mathrm{~L} \mathrm{~min}^{-1}$ (a slight overpressure was maintained in the detector vessel). This mode of operation (continuous flushing) was used for all measurements of ${ }^{222} \mathrm{Rn}$ content in sampled gases, therefore the obtained here count rates are 


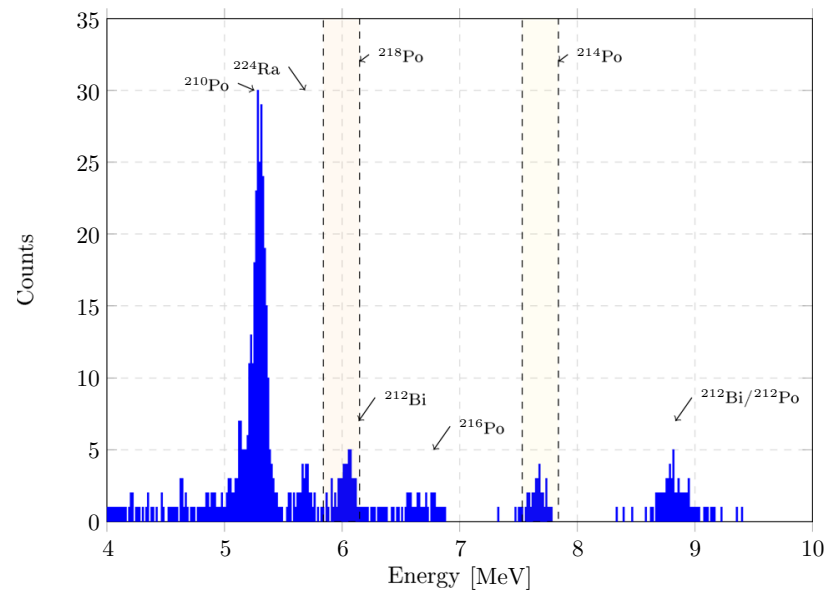

Fig. 9 Background spectrum accumulated over $24.8 \mathrm{~d}$, the detector vessel was flushed with $\mathrm{HPN}_{2}$ at $5 \mathrm{~L} \mathrm{~min}^{-1}$ (after application of the PSD)

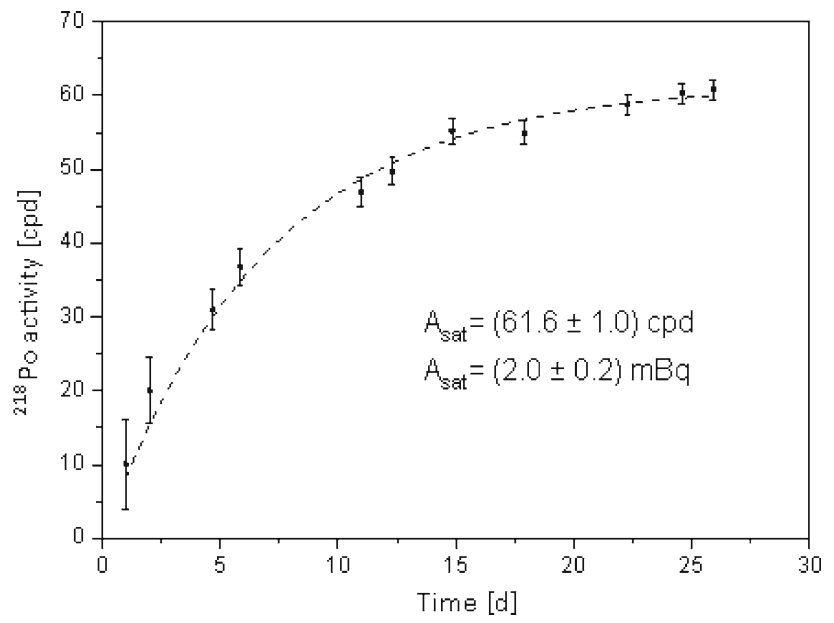

Fig. $10{ }^{218}$ Po count rate as a function of time for the detector vessel filled with $\mathrm{HPN}_{2}$ and closed. The fitted curve corresponds to the ${ }^{222} \mathrm{Rn}$ in-growth with the half-life of $3.8 \mathrm{~d}$, as observed over almost 4 weeks

used as the reference values of the detector background. Sampling of the clean room air was carried out at the flow rate of approximately $15 \mathrm{~L} \mathrm{~min}^{-1}$ and pressure inside the vessel of around 915 mbar. The flow rate was determined by the maximum allowed pressure in the vessel (defined by the diameter of the exhaust pipe, construction of the vessel, gas flow regulator parameters etc.). In order to minimize the activity build-up from the detector self-emanation and to maximize air mixing in the chamber we had to maximize the airflow.

The cumulative spectrum acquired over $24.8 \mathrm{~d}$ is shown in Fig. 9. Clearly the ${ }^{210}$ Po peak dominates $\left({ }^{210} \mathrm{Po}\right.$ accumulated on the detector surface from the air exposures and from the previous tests with ${ }^{222} \mathrm{Rn}$ sources). Beside ${ }^{218} \mathrm{Po}$ and ${ }^{214} \mathrm{Po}$, peaks are also visible from the ${ }^{220} \mathrm{Rn}$ decay chain. The registered ${ }^{220} \mathrm{Rn}$ and ${ }^{222} \mathrm{Rn}$ cannot come from the high purity nitrogen $\left({ }^{222} \mathrm{Rn}\right.$ concentration in $\mathrm{HPN}_{2}$ is below $1 \mu \mathrm{Bq} \mathrm{m}^{-3}$, thus not measurable by this monitor), but from internal emanation and imperfect flushing with $\mathrm{HPN}_{2}$. The measured count rates in the energy regions for ${ }^{218} \mathrm{Po},{ }^{214} \mathrm{Po}$ and ${ }^{210} \mathrm{Po}$ were $(6.4 \pm 0.5) \mathrm{cpd},(3.0 \pm 0.4) \mathrm{cpd}$ and $(59.9 \pm 1.6) \mathrm{cpd}$, respectively.

\subsubsection{Sensitivity}

The sensitivity of the detector can be discussed in terms of the minimum measurable activity (MMA) [4]. It is defined as the activity measurable with a given instrument for assumed a priori precision. Following the formalism presented in $[2,20]$, MMA can be calculated from the measured background count rate $B$ for a given isotope (in our case ${ }^{214} \mathrm{Po}$ ) and for the known detection efficiency $\epsilon$. The minimum measurable activity $\left(A_{0}\right)$ can be evaluated starting from the formula expressing the final relative uncertainty of the measurement:

$\delta=\frac{\sigma_{A_{0}}}{A_{0}}$

where $\sigma_{A_{0}}$ is the standard deviation of $A_{0}$, and $\delta$ is the assumed relative uncertainty. $A_{0}$ and $\sigma_{A_{0}}$ can be expressed by the total number of ${ }^{214} \mathrm{Po}$ counts $N$ registered in the counting time interval $\Delta t$, including also the background count rate $B$ :

$N=\epsilon A_{0} \Delta t+B \Delta t$

Calculation of $\sigma_{A_{0}}$ takes into account the uncertainties of the detector background $\sigma_{B}$ and the uncertainty of the detection efficiency $\sigma_{\epsilon}$.

The final formula for $A_{0}$ is the following:

$A_{0}=\frac{1+\sqrt{1+4\left(\Delta t^{2} \sigma_{B}^{2}+B \Delta t\right)\left(\delta^{2}-\delta_{\epsilon}^{2}\right)}}{2 \epsilon \Delta t\left(\delta^{2}-\delta_{\epsilon}^{2}\right)}$

where $\delta_{\epsilon}=\sigma_{\epsilon} / \epsilon$.

Taking into account that $C_{\min }=A_{0} / V$, where $V$ is the volume of the detector vessel and the parameters determined above for ${ }^{214} \mathrm{Po}$, requiring $\delta=0.3$ and assuming $\Delta t=24 \mathrm{~h}$ $\left({ }^{222} \mathrm{Rn}\right.$ concentration monitored in 1-d bins) one can find that the minimum measurable specific ${ }^{222} \mathrm{Rn}$ activity for our detector is $C_{\text {min }}=1.1 \mathrm{mBq} \mathrm{m}^{-3}$.

One should note here that the obtained $C_{\min }$ is not a detection limit - this is still a number with a specified a priori uncertainty. For calculation of the latter we adopt the formula from [6] (to be able to compare directly different detectors). The 90\% confidence level (CL) detection limit $L_{C}$ is given as:

$L_{C}=\frac{1.64 \times \sigma_{B}}{C F}$ 
Table 1 Parameters of the DARKSIDE online radon monitor

\begin{tabular}{|c|c|c|}
\hline Parameter & Value & Remarks \\
\hline $\begin{array}{l}\text { Detection efficiency } \\
\epsilon\end{array}$ & $(36 \pm 3) \%$ & $\begin{array}{l}\text { Absolute detection } \\
\text { efficiency } \\
\text { measured for the } \\
\text { Ateko air for }{ }^{218} \mathrm{Po} \\
\text { and }{ }^{214} \mathrm{Po} \text { at } \mathrm{HHV} \\
=8 \mathrm{kV}\end{array}$ \\
\hline \multirow[t]{2}{*}{ Background $B$} & $(6.4 \pm 0.5) \mathrm{cpd}^{218} \mathrm{Po}$ & $\begin{array}{l}\text { Detector vessel } \\
\text { continuously } \\
\text { flushed with } \mathrm{HPN}_{2} \\
\left(5 \mathrm{~L} \mathrm{~min}^{-1}\right)\end{array}$ \\
\hline & $\begin{array}{l}(3.0 \pm 0.4) \mathrm{cpd}^{214} \mathrm{Po} \\
(59.9 \pm 1.6) \mathrm{cpd}^{210} \mathrm{Po}\end{array}$ & \\
\hline $\begin{array}{l}\text { Internal }{ }^{222} \mathrm{Rn} \\
\text { emanation of the } \\
\text { detector } \mathrm{A}_{\text {sat }}\end{array}$ & $(2.0 \pm 0.2) \mathrm{mBq}$ & Saturation value \\
\hline$C_{\min }$ & $1.1 \mathrm{mBq} \mathrm{m}^{-3}$ & $\begin{array}{l}\text { Assuming } 30 \% \\
\text { accuracy }\end{array}$ \\
\hline Detection limit & $0.05 \mathrm{mBq} \mathrm{m}^{-3}$ & At $90 \%$ C.L. \\
\hline
\end{tabular}

where $C F\left[\mathrm{cpd} /\left(\mathrm{mBq} / \mathrm{m}^{3}\right)\right]$ is a calibration factor defined as a ratio of the registered count rate to the real radon concentration in the detector. In our case $C F=13.9 \mathrm{cpd} /\left(\mathrm{mBq} / \mathrm{m}^{3}\right)$ and $\sigma_{B}=0.4 \mathrm{cpd}$, thus $L_{C}=0.05 \mathrm{mBq} \mathrm{m}^{-3}$.

For the fixed $\delta=0.3$ one can still improve (lower) the $C_{\min }$ below $1 \mathrm{mBq} \mathrm{m}^{-3}$ by reducing e.g. the detector background (additional cleaning of the detector vessel, higher $\mathrm{HPN}_{2}$ flow rate) or by improving the detection efficiency. As discussed earlier, in the present geometry by increasing HHV up to e.g. $15 \mathrm{kV}$, it is possible to reach $\epsilon \approx 0.41-0.42$. With a simultaneous detector background reduction by a factor of e.g. 2 one could get $C_{\min } \approx 0.7 \mathrm{~m} \mathrm{~Bq} \mathrm{~m}^{-3}$, a $36 \%$ improvement over the current value of $1.1 \mathrm{~m} \mathrm{~Bq} \mathrm{~m}^{-3}$. Correspondingly, the detection limit would also improve.

The most important parameters of the detector are collected in Table 1.

\section{Results}

Radon concentration has been measured sequentially from the air immediately at the output of the Ateko radon abatement system (Ateko air) and the air from both clean rooms following the installation of the radon monitor. Selected results are collected in Table 2.

As the first sample we measured the air taken directly from the radon abatement system shortly after its installation at LNGS. The test gave $(15 \pm 2) \mathrm{mBq} \mathrm{m}^{-3}$. After some upgrades of the plant, another measurement was performed and yielded $(1.4 \pm 0.4) \mathrm{mBq} \mathrm{m}^{-3}$. At the same time the Alpha Guard instrument monitoring ${ }^{222} \mathrm{Rn}$ content in Hall

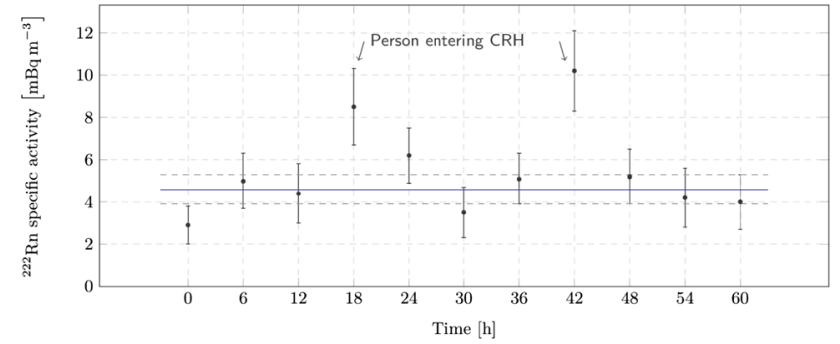

Fig. 11 An example of the ${ }^{222} \mathrm{Rn}$ content monitoring in the CRH clean room over a weekend, evaluated every $4 \mathrm{~h}$. $\mathrm{CRH}$ was generally unoccupied. The two highest values shown in the plot were recorded after a person entered the room for about 1 hour. After excluding these two measurements the average specific activity of the clean room was $(4.6 \pm 0.6) \mathrm{mBq} \mathrm{m}^{-3}$ (horizontal lines)

$\mathrm{C}$ showed about $50 \mathrm{~Bq} \mathrm{~m}^{-3}$. The achieved radon reduction factor was thus $\approx 3.6 \times 10^{4}$.

Measurements of the air sampled from CRH were also performed in parallel. The measured activities of ${ }^{222} \mathrm{Rn}$ ranged between 6 and $36 \mathrm{mBqm}^{-3}$. Activity of personnel working inside the clean room was clearly visible as an increased ${ }^{222} \mathrm{Rn}$ content in the sampled air. An example of such events is shown in Fig. 11. The reported ${ }^{222} \mathrm{Rn}$ content for the clean room reflects the total activity, including also ${ }^{222} \mathrm{Rn}$ introduced by the personnel.

Measurements performed on samples taken from CR1 yielded between $200 \mathrm{mBq} \mathrm{m}^{-3}$ and $5100 \mathrm{mBq} \mathrm{m}^{-3}$, clearly higher compared to the values obtained for CRH. The volumes of the clean rooms are comparable (the CR1 surface is $62 \mathrm{~m}^{2}$ and its volume approximately $186 \mathrm{~m}^{3}$, the CRH surface is $38 \mathrm{~m}^{2}$ and its volume approximately $208 \mathrm{~m}^{3}$ ). The higher ${ }^{222} \mathrm{Rn}$ concentration in CR1 may be related to its positioning directly on the floor of Hall C causing some emanation through imperfect seals of the floor panels (for reference, $\mathrm{CRH}$ is not in contact with the floor or wall of the underground cavern). In addition, CR1 experienced a $\sim 10$-fold increase in radon concentration in 2016-17 with respect to 2014. This increase might be ascribed to a higher accumulation of equipment inside it, general contamination of the clean room once DARKSIDE-50 was installed and running, and personnel activities at different time periods during the 5 years of continuous measurements.

\section{Summary}

We have installed and commissioned an electrostatic ${ }^{222} \mathrm{Rn}$ detector able to monitor the radon content in air at the output of the Ateko radon abatement system and sampled from CRH and CR1 operated by the DARKSIDE-50 Collaboration. The care in design and construction allowed to reach the minimum measurable specific activity of $1.1 \mathrm{mBq} \mathrm{m}^{-3}$ and a record detection limit of about $0.05 \mathrm{mBq} \mathrm{m}^{-3}$. The mea- 
Table 2 Radon concentrations in different air (and nitrogen) samples measured with the electrostatic detector over 5 years of continuous operation. Consecutive measurements correspond to different periods of time, and are characterized by varying level of activity by personnel in the clean rooms

\begin{tabular}{|c|c|c|}
\hline Sample & Date & ${ }^{222} \mathrm{Rn}$ content $\left(\mathrm{mBq} \mathrm{m}^{-3}\right)$ \\
\hline \multirow[t]{4}{*}{ Ateko air } & July 2013 & $15 \pm 2$ \\
\hline & November 2013 & $1.4 \pm 0.4$ \\
\hline & September 2014 & $11 \pm 1$ \\
\hline & March 2017 & $4.9 \pm 2.2$ \\
\hline \multirow[t]{6}{*}{ CRH air } & July 2013 & $21 \pm 3$ \\
\hline & October 2013 & $12 \pm 2$ \\
\hline & November 2013 & $5.8 \pm 0.9$ \\
\hline & September 2014 & $36 \pm 6$ \\
\hline & October 2016 & $19 \pm 4$ \\
\hline & March 2017 & $10.6 \pm 3.2$ \\
\hline \multirow[t]{3}{*}{ CR1 Air } & November 2013 & $208 \pm 21$ \\
\hline & October 2016 & $5100 \pm 100$ \\
\hline & March 2017 & $2500 \pm 100$ \\
\hline \multirow{3}{*}{$\begin{array}{l}\text { High purity nitrogen } \\
\left(\mathrm{HPN}_{2}\right)^{\mathrm{a}}\end{array}$} & November 2013 & $0.5 \pm 0.1$ \\
\hline & September 2014 & $0.7 \pm 0.2$ \\
\hline & March 2017 & $3.5 \pm 2.0$ \\
\hline Hall C Air & $\begin{array}{c}\text { November } 2013 \\
\text { Measured with } \\
\text { Alpha Guard }\end{array}$ & $\approx 50 \mathrm{~Bq} \mathrm{~m}^{-3}$ \\
\hline
\end{tabular}

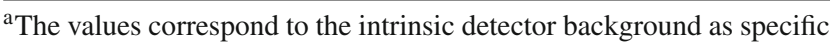
${ }^{222} \mathrm{Rn}$ concentration of $\mathrm{HPN}_{2}$ is below $1 \mu \mathrm{Bqm}^{-3}$

surements performed showed that the DS-50 radon abatement system reduces the radon content in the Hall-C air by a factor of $3.6 \times 10^{4}$ (comparable to the desired $10^{5}$ ). Air taken from CRH and CR1 during the installation of DS-50 in 2013 showed on average $10 \mathrm{mBq} \mathrm{m}^{-3}$ and $200 \mathrm{mBq} \mathrm{m}^{-3}$, respectively. Both clean rooms were operated with the lowest ever reported ${ }^{222} \mathrm{Rn}$ concentrations (especially $\mathrm{CRH}$ ), comparable with small clean benches flushed with nitrogen gas. The electrostatic radon monitor was one of the factors that allowed to successfully carry out the research program of DS50 [21-25]. The experience matured with the low-radon procedures for the preparation, assembly, and deployment of the DARKSIDE-50 TPC detector will be valuable when applied to the scaled-up, multi-ton successor experiment, DARKSIDE-20k [26] and other forthcoming ultra-low background experiments.

Acknowledgements This work was supported in part by the Polish National Science Centre (Grant no. UMO-2012/05/E/ST2/02333), the Foundation for Polish Science (Grant no. POIR.04.04.00-00-2FFF/16), and the US National Science Foundation (Grant PHY-1004072). The detector hardware was acquired in large part through US NSF Grant PHY-1242585. We acknowledge the help of Nicola Canci and Peter
Meyers in significantly improving the manuscript, and the Laboratory Nazionali del Gran Sasso for their hospitality.

Data Availability Statement This manuscript has no associated data or the data will not be deposited. [Authors' comment: As for the data availability, the only data relevant to the publication is present in form of published graphs or tables. No database is associated with the article that could be shared.]

Open Access This article is licensed under a Creative Commons Attribution 4.0 International License, which permits use, sharing, adaptation, distribution and reproduction in any medium or format, as long as you give appropriate credit to the original author(s) and the source, provide a link to the Creative Commons licence, and indicate if changes were made. The images or other third party material in this article are included in the article's Creative Commons licence, unless indicated otherwise in a credit line to the material. If material is not included in the article's Creative Commons licence and your intended use is not permitted by statutory regulation or exceeds the permitted use, you will need to obtain permission directly from the copyright holder. To view a copy of this licence, visit http://creativecomm ons.org/licenses/by/4.0/.

Funded by $\mathrm{SCOAP}^{3}$.

\section{References}

1. G. Zuzel, H. Simgen, High sensitivity radon emanation measurements. Appl. Radiat. Isot. 67, 889-893 (2009)

2. M. Wójcik, G. Zuzel, A high sensitivity large volume cryogenic detector for ${ }^{222} \mathrm{Rn}$ in gas. J. Radioanal. Nucl. Chem. 277, 199-205 (2008)

3. M. Wójcik, G. Zuzel, H. Siemgen, Review of high-sensitivity radon studies. Int. J. Mod. Phys. A 32(30), 1743004 (2017)

4. M. Wójcik, W. Wlazło, A high sensitivity scintillation chamber for radon in gas. Nucl. Instrum. Methods A 345(2), 351-355 (1994)

5. Y. Takeuchi et al., Development of high sensitivity radon detectors. Nucl. Instrum. Methods A 421, 334-341 (1999)

6. C. Mitsuda et al., Development of super-high sensitivity radon detector for the super-Kamiokande detector. Nucl. Instrum. Methods A 497, 414-428 (2003)

7. J. Kiko, Detector for ${ }^{222} \mathrm{Rn}$ measurements in air at the $1 \mathrm{mBqm}^{-3}$ level. Nucl. Instrum. Methods A 460(2), 272-277 (2001)

8. A. Pocar, Low background techniques and experimental challenges for Borexino and its nylon vessels. Ph.D. Dissertation, Princeton University (2003)

9. J. Benziger et al., The nylon scintillator containment vessel for the Borexino solar neutrino experiment. Nucl. Instrum. Methods A 582, 509-534 (2007)

10. P. Agnes et al., (The DarkSide Collaboration), The veto system of the DarkSide-50 experiment. J. Instrum. 11, P03016 (2016)

11. A.S. Ateko, Radon Reduction System. http://www.ateko.cz. Accessed 6 July 2020

12. G. Benato et al., Radon mitigation during the installation of the CUORE $0 \nu \beta \beta$ decay detector. J. Instrum. 13, P01010 (2018)

13. K. Pelczar, Mobility and lifetime of ${ }^{220} \mathrm{Rn}$ daughters. AIP Conf. Proc. 1921, 090003 (2018)

14. P. Dular, C. Geuzaine, GetDP reference manual: the documentation for GetDP, a general environment for the treatment of discrete problems. http://www.getdp.info. Accessed 6 July 2020

15. K. Pelczar, Backgrounds in the Gerda and DarkSide experiments. Radioactive Ions in cryogenic liquids. Ph.D. Dissertation, Jagiellonian University (2016) 
16. G. Heusser et al., ${ }^{222} \mathrm{Rn}$ detection at the $1 \mathrm{mBqm}^{-3}$ range in nitrogen gas and a new Rn purification technique for liquid nitrogen. Appl. Radiat. Isot. 52(3), 691-695 (2000)

17. J. Kiko, Noise reduction for proportional counter signals in the GNO solar neutrino experiment. Nucl. Instrum. Methods A 482, 434-440 (2002)

18. P.K. Hopke, Use of electrostatic collection of ${ }^{218}$ Po for measuring Rn. Health Phys. 57, 39-42 (1989)

19. P. Pagelkopf, J. Porstendörfer, Neutralisation rate and the fraction of the positive ${ }^{218}$ Po-clusters in air. At. Environ. 37, 1057-1064 (2003)

20. M. Misiaszek et al., Optimization of low-background alpha spectrometers for analysis of thick samples. Appl. Radiat. Isot. 81, 146$150(2013)$

21. P. Agnes et al., (The DarkSide Collaboration), First results from the DarkSide-50 dark matter experiment at Laboratori Nazionali del Gran Sasso. Phys. Lett. B 743, 456-466 (2015)

22. P. Agnes et al., (The DarkSide Collaboration), Results from the first use of low radioactivity argon in a dark matter search. Phys. Rev. D 93, 081101 (2016)
23. P. Agnes et al., (The DarkSide Collaboration), DarkSide-50 532 day Dark Matter Search with Low-Radioactivity Argon. Phys. Rev. D 98, 102006 (2018)

24. P. Agnes et al., (The DarkSide Collaboration), Low-mass dark matter search with the DarkSide-50 Experiment. Phys. Rev. Lett. 121, 081307 (2018)

25. P. Agnes et al., (The DarkSide Collaboration), Constraints on SubGeV Dark Matter-Electron Scattering from the DarkSide-50 Experiment. Phys. Rev. Lett. 121, 111303 (2018)

26. C.E. Aalseth et al., Darkside-20k: a 20 tonne two-phase LAr TPC for direct dark matter detection at LNGS. Eur. Phys. J. Plus 133, 131 (2018) 